\section{LETTERS TO THE EDITORS}

The Editors do not hold themselves responsible for opinions expressed by their correspondents. No notice is taken of anonymous communications

\section{Development of the Universe}

The theories of Mr. F. Hoyle and Prof. Pascual Jordan have renewed an interest in an important cosmological problem: How and when did the substance of the material universe come into existence ? It may facilitate discussion if the difficulties in the way of the various theories about this are summarized in the simplest possible terms.

The difficulty in the theory that all the matter in the universe has existed for an infinite time is that it would then have reached certain limiting conditions, limits, for example, of concentration; of entropy; of radius of curvature of an expanding universe. Mathematical analysis has shown that no such limits have been reached.

The difficulty in the assumption that all the matter in the universe has existed for a finite time is of a different kind. The assumption depends on the hypothesis that two moments in time have had a peculiar significance. One is the moment when matter first began to appear, the other the moment when the process of appearance stopped. This assumption is further based on the hypothesis that, while holding rigidly now in such a way as to cause the total energy in the universe to be constant, the principle of conservation of energy did not hold in the same way during the period between the two above-mentioned hypothetical moments of time.

One of several difficulties in Prof. Jordan's theory is the underlying hypothesis that $10^{80}$ protons can have a real existence for a definite period of time before, in the form of a star, they emit a signal by which they could be detected. Mr. Hoyle's theory, though it has the advantage of simplicity, raises one grave difficulty. If interstellar space had been witnessing the appearance of matter for an infinitely long time, an infinite amount of it would have followed a gravitational field in the direction of such large accumulations as the nebulæ. So these would have reached infinite size.

This latter difficulty would be overcome if one assumes that the continuous appearance of matter is accompanied by its continuous disappearance; though both proeesses noed not be at the same rate. If so, the present increase in the total contents of the universe postulated both by Hoyle and Jordan is a differential effect. This assumption contains, $I$ think, a minimum of hypothesis. It does not postulate the simultaneous appearance of any specific or even large numbers of protons, and allows for the possibility that single small particles may appear at any given time or place. It does not postulate that any given region in space is more privileged than any other to be the site of the appearance of a new particle, and it does not postulate that any existing particle is more privileged than any other to disappear at a given moment.

The automatie results of such completely random appearance and disappearance would be that most new matter would come into existence in interstellar space, while most disappearances would occur in concentrations such as the nebulæ. Thus these would be continuously renewed and roughly compensated for the matter that has fallen, as it were, through them out of existence.
If matter is both appearing and disappearing at all times completely at random, the second Law of Thermodynamics will not lead to an infinite increase in entropy, for the increase will be counteracted by the potential energy of new matter in interstellar space. The net increase need not always be positive. It could sometimes be negative and cause a reversal of the present expansion of the universe. While this was happening, the total mass of nebulæ as well as individual stars would tend to diminish, and it should be possible to show that there is a maximum concentration above which disappearances are more rapid than appearances, so that concentrations of matter can only vary between specific limits.

Lastly, there appears to be some misapprehension concerning the bearing of the principle of conservation of energy on cosmological theories. This principle states that in a self-contained system the total amount of energy is constant. If matter may appear or disappear at random, the conclusion is that, in practice, one cannot be sure of ever ha,ving a selfcontained system, not that the principle does not hold.

University College,

REGINALD O. KAPP London, W.C.1. Oct. 21.

Is the system of cosmology I have advocated, and also in the parallel theory of Bondi and Gold, the nebulæ are not all of infinite age as Prof. R. O. Kapp seems to imagine. In a sufficiently large volume $V \mathrm{~cm}^{8}$ of space, the number of nebulæ with ages greater than $T$ ( $T$ being measured in units of Hubble's constant, namely, $1.8 \times 10^{8}$ years) is about $10^{-72} \mathrm{~V}$ $e^{-3 T}$, the average spacing between nebulæ older than $T$ being about $10^{24} e^{T} \mathrm{~cm}$. By taking $V$ large enough, it is true that a nebula of arbitrarily large $T$ can be found. But this has little interest when related to astronomical observation, for the effect of the red shift of light, arising from the expansion of the universe, is to make the observation of events occur. ring at distances greater than about $1.7 \times 10^{27} \mathrm{~cm}$. inherently impossible (even for an observer equipped with a perfect telescope). It follows, therefore, that the oldest nebulæ within the observable portion of the universe have ages $T$ given, so far as order of magnitude is concerned, by

$$
10^{24} \exp T=1.7 \times 10^{27}
$$

that is, $T=7.44$ Hubble units $=1.34 \times 10^{10}$ years. These nebulæ have been identified tentatively with the nebulæ in the great clusters. It may be noted in passing that a specially chosen observer can detect one condensation having an age $T$ appreciably greater than the value given by the above equation. But the probability of this occurring for an observer taken at random decreases with increasing $T$ according to the factor exp $-3 T$.

The above remarks summarize results which show that, although condensations of increasing age and size can occur, their distances from the observer in. crease correspondingly. This has the effect of making very old nebulæ optically unobservable, and also of making negligible their influence on the metric in the portion of the universe accessible to the observer.

It appears, therefore, that Prof. Kapp's objection concerns a question that has no relation to observa. tion. A similar remark applies to an issue raised by Prof. Jordan in Nature of October 15, 1949, p. 637. He points out that my work leads to the conclusion that there must be an inter-nebular medium in which 